\title{
Leveraging Phone Based Mobile Technology to Improve Data Quality at Health Facilities in Rural Malawi: A Best Practice Project
}

\section{Tinashe Alinafe Tizifa ( $\nabla$ t.tizifa@amsterdamumc.nl )}

University of Malawi College of Medicine https://orcid.org/0000-0001-5040-2807

\section{William Nkhono}

University of Malawi College of Medicine

\section{Spencer Mtengula}

University of Malawi College of Medicine

Michele van Vugt

Academisch Medisch Centrum: Amsterdam UMC Locatie AMC

\section{Zachary Munn}

JBI: The University of Adelaide Joanna Briggs Institute

Alinune Nathanael Kabaghe

University of Malawi College of Medicine

\section{Research}

Keywords: Malaria, Electronic data capture, Information systems, Evidence-Based Implementation, Baseline audit, GRIP matrix, Endline audit

Posted Date: December 2nd, 2020

DOI: https://doi.org/10.21203/rs.3.rs-116120/v1

License: (1) This work is licensed under a Creative Commons Attribution 4.0 International License. Read Full License 


\section{Abstract}

Background: To further reduce malaria burden, identification of areas with highest burden for targeted interventions needs to occur. Routine health information has the potential to indicate where and when clinical malaria occurs the most. Developing countries mostly use paper-based data systems however they are errorprone as they require manual aggregation, tallying and transferring of data. We piloted the use of electronic data capture (EDC) using a cheap and user friendly software in rural Malawian primary healthcare setting to improve the quality of health records.

Methodology: We used audit and feedback tools from Joanna Briggs Institute's (Practical Application of Clinical Evidence System and Getting Research into Practice) in four primary healthcare facilities. Using this approach, we identified the best available evidence for a malaria information system (MIS). Baseline audit of the existing MIS was conducted in the facilities based on available best practice for MIS; this included ensuring data consistency and completeness in MIS by sampling 25 random records of malaria positive cases. Implementation of an adapted evidence-based EDC system using tablets on an OpenDataKit platform was done. We then conducted an end line audit following implementation. Users had interviews on experiences and challenges concerning EDC at the beginning and end of the survey.

Results: The existing MIS was paper-based, occupied huge storage space, had some data losses due to torn out papers and were illegible in some facilities. The existing MIS did not have documentation of necessary parameters such as malaria deaths and treatment within 14 days. Training manuals and modules were absent. One health center solely had data completeness and consistency at $100 \%$ of the malaria-positive sampled records. Data completeness and consistency rose to $100 \%$ with readily available records containing information on recent malaria treatment. Interview findings at the end of the survey showed that EDC was acceptable among users and they agreed that the tablets and the OpenDataKit were easy to use, improved productivity and quality of care.

Conclusions: Improvement of data quality and use in the Malawian rural facilities was achieved through the introduction of EDC using OpenDataKit. Health workers in the facilities showed satisfaction with the use of EDC. (Word limit: 350 words, current: 349)

\section{Introduction}

Malaria was responsible for 219 million cases and 435,000 deaths in 2017 with more than $90 \%$ of both cases and deaths occurring in Africa (1). In both low and high transmission settings, there are geographical and seasonal variations of malaria burden (2). Individual, household, community and environmental factors affect the distribution of malaria infection. Identifying and addressing these variations may inform targeted interventions to reduce the burden of malaria in affected communities.

The World Health Organization's (WHO) Global Technical Strategy for Malaria 2016-30 (GTS) for malaria control suggests the transformation of malaria surveillance into a core intervention which would allow the capture of essential malaria data on a continuous basis thereby improving response to outbreaks, assessment of disease trends and optimization of responses (3). This pillar further explains that surveillance of malaria should be utilized to inform appropriate action (3). Strong surveillance systems linked to effective responses 
are critical for malaria elimination $(4,5)$. The process of data collection together with reporting, active case finding, and linkage to response must be swift in endemic settings for proper identification of infections, prevention of ongoing transmission, and decrease transmission efficiency of vectors $(4,5)$. In a majority of sub-Saharan African (SSA) countries, routine malaria surveillance involves collecting and reporting aggregate data from public health facilities through the national health management information systems (HMIS) (6). Generally, malaria burden is monitored using household surveys or routinely collected health information. Household surveys are costly to conduct and analyze and are usually based on a cross sectional design in a proportion of the population (7). The cross sectional design does not allow monitoring of burden over time.

Data collected in health facility registers for patients tested for malaria are valuable for monitoring trends in malaria morbidity and evaluating the impact of malaria control interventions (8). However, the data may be incomplete, unreliable and paper-based and therefore prone to errors during manual tallying (6). In addition, facilities may use several registers for a single patient making it complex to easily access individual patient information(9). When it comes to reporting from primary health-care facilities to secondary health-care facilities, these data are aggregated by test result and age category; the aggregated reports lose valuable information including its location.

Most health facilities in Malawi use paper-based registers to record and report routine malaria information. The paper-based registers are also used to record other illnesses at the facility. For each illness, the records are tallied and compiled into an aggregated report which is physically sent to the district health office. The reports from all health facilities in the district are entered into the district health information system (DHIS) using a computer. This system, as already elaborated above presents a number of challenges more especially with errors that have not been addressed during primary data collection.

Malawi's national malaria operational plan includes goals to improve the country's capacity to collect and use information and to adapt to changing epidemiology and incorporate new tools by 2020 (10). For monitoring progress towards achievement of these goals, a system for capturing both local and regional transmission is essential. Particularly, there is a need for an effective surveillance system that can monitor near real-time malaria data, target focal areas of infection, increase capacity to identify transmission hotspots and rapidly identify changes in malaria transmission, morbidity and mortality.

In this paper, we describe an evidence-based quality improvement project for a malaria information system conducted at four health facilities in a rural community of Chikhwawa in Southern Malawi. The overall goal was to develop and deploy an evidence based best practice system to improve the quality and use of the health information system. We also discuss lessons learned and experiences related to implementation of the system.

\section{Methodology}

\section{Study design}

We followed the JBI approach to evidence implementation(11) including the use of the Practical Application of Clinical Evidence System (PACES) software(11) and Getting Research into Practice (GRiP) framework for 
promoting evidence based health care following attendance at an evidence implementation training program, funded through a competitive scholarship(12-14). This approach involves three phases of activity:

1. Establishing a team for the project and undertaking a baseline audit based on criteria informed by the evidence.

2. Reflecting on the results of the baseline audit, providing feedback and designing and implementing strategies to address non-compliance found in the baseline audit informed by the JBI GRiP framework

3. Conducting a follow up audit to assess the outcomes of the interventions implemented to improve practice, and identify future practice issues to be addressed in subsequent audits.

\section{Project site}

The project was conducted from September 2018 to January 2019 in 4 health facilities namely Chapananga, Kakoma, Kapichira and Majete 1 in the southern Malawi district of Chikhwawa. The facilities are situated within the catchment area of the Majete wildlife reserve (MWR). This project site has been previously described (15) but briefly the catchment area is characterized by highly heterogeneous land use for subsistence farming and small scale business. Chikhwawa has a hot and humid tropical climate for most of the year. Some of the services offered in these facilities include out-patient department (OPD), maternity, under-five clinic and immunization.

\section{Ethical considerations}

The project was registered as a quality improvement activity within the health centers, and therefore did not require ethical approval. Approval to conduct the project in Chikhwawa district was however provided by Chikhwawa District Health Office.

\section{Data collection}

The WHO established standard protocols for monitoring and surveillance of malaria treatment, both in children and adults, specifically from low and middle-income countries $(\mathrm{LMIC})(16,17)$ informed our approach. We explored the literature to obtain evidence on the best available practice for electronic monitoring of the quality of malaria treatment in children living in LMIC which was synthesized in an evidence summary and informed the criteria development. The data collection process was categorized into four phases, with these phases progressing in sequential order.

\section{Phase 1: Team establishment and baseline audit}

The first phase of the project was to form a core project team to provide technical, logistical and strategic guidance. During this phase, a team to coordinate all project activities was established and comprised of health care workers (HCW) from Chikhwawa district health office (DHO), particularly data clerks and medical assistants. These are based at the implementing health center. Some members from the DHO that were incorporated include the District Medical Officer (DMO) and the health management information system (HMIS) officer. Others team members included information technology specialists, masters' student, PhD student and a postdoctoral researcher all working with the College of Medicine, University of Malawi. DHO personnel provided support in engagement of the health centers. Health center personnel were to be the implementers of the project. The composition and roles of personnel are summarized in (appendix 1). 
After forming the team, audit criteria were developed (Table 1). A structured questionnaire was created and administered to the health facility in-charges (usually medical assistants) and data clerks to assess the existing health facility information system. Furthermore, documentation of roles and responsibilities of personnel at each level of the data monitoring system was checked. We asked about the existence of training manuals and modules for the monitors together with the type of infrastructure at each facility.

In order to determine prior treatment of malaria within 14 days, a checklist was also developed for OPD, laboratory and antimalarial registers. In addition, records of 25 randomly selected malaria positive patients in each facility were checked for completeness and internal consistency. Completeness was assessed by checking that all required data elements had a recording while consistency was assessed by comparing the data in all the three registers. This audit required a total of two days with a maximum of three hours at each site for it to be completed.

Table 1

Shows the evidence informed audit criteria used in the project (baseline and follow-up audit) together with a description of the sample and approach to measuring compliance with best practice for each audit criterion.

\begin{tabular}{|c|c|c|}
\hline Audit criterion & Sample & Method to measure \\
\hline $\begin{array}{l}\text { 1. eMIS is used for quick access to } \\
\text { both individual and aggregated data. }\end{array}$ & 4 health facilities & Interview health facility manager \\
\hline $\begin{array}{l}\text { 2. MIS includes suspected, tested and } \\
\text { confirmed cases }\end{array}$ & 4 health facilities & $\begin{array}{l}\text { Checklist for OPD, laboratory and } \\
\text { Artemisinin-Lumefantrine }(A L) \text { registers }\end{array}$ \\
\hline $\begin{array}{l}\text { 3. Monitoring system includes location } \\
\text { of information }\end{array}$ & 4 health facilities & Checklist of OPD register \\
\hline $\begin{array}{l}\text { 4. Monitoring system includes prior } \\
\text { treatment (within } 14 \text { days) }\end{array}$ & 4 health facilities & Checklist of OPD register \\
\hline $\begin{array}{l}\text { 5. Monitoring system includes malaria } \\
\text { treatment and dose information }\end{array}$ & 4 health facilities & $\begin{array}{l}\text { Checklist for OPD \& antimalarial } \\
\text { registers }\end{array}$ \\
\hline $\begin{array}{l}\text { 6. Data in monitoring system is } \\
\text { complete }\end{array}$ & $\begin{array}{l}25 \text { malaria case } \\
\text { records at each } \\
\text { facility }\end{array}$ & $\begin{array}{l}\text { Check retrospective records in OPD, } \\
\text { laboratory and antimalarial registers }\end{array}$ \\
\hline $\begin{array}{l}\text { 7. Internally consistent with no } \\
\text { duplications in monitoring system }\end{array}$ & $\begin{array}{l}25 \text { malaria case } \\
\text { records at each } \\
\text { facility }\end{array}$ & $\begin{array}{l}\text { Check retrospective records in OPD, } \\
\text { laboratory and antimalarial registers }\end{array}$ \\
\hline $\begin{array}{l}\text { 8. Roles, responsibilities, training } \\
\text { manuals \& modules available }\end{array}$ & 4 health facilities & Interview health facility manager \\
\hline
\end{tabular}

\section{Phase 2: Design and implementation of strategies to improve practice: GRiP strategy}

After obtaining the baseline audit results, the project team identified strategies that would help address the bottlenecks in data management. The team developed an electronic case report form (eCRF) based on the existing malaria, mRDT and OPD registers ensuring that the eCRF (appendix 2) was a digital replica of the paper based forms (appendix 3). The eCRF was designed using (Extensible Markup Language) XML script, accounting for all the variables which were on the paper-based register and available evidence for electronic 
monitoring of the quality of malaria treatment in children in low and middle-income countries. Testing of the eCRF was first done by the project team, to ensure that all skip logic functioned, and all the required data and variables were being collected by the electronic form. In order for data collection to occur at the health centers, Samsung tablets from Majete malaria project were installed an application called Open Data Kit (ODK); the eCRF was uploaded and configured to run as a client for data collection on these Samsung tablets (appendix 4).

ODK is an open source android application which can be used to capture disaggregated individual records using mobile devices; the records can be sent remotely to a server or desktop and is immediately accessible using a specific Uniform Resource Locator (URL) and appropriate log in credentials. ODK can collect text, numeric data, images and geographical coordinates in resource limited settings by people with minimal education qualifications(18). ODK can run both on Windows machines and Android tablets, however, android tablets were selected because they have superior battery life and require minimal technical knowledge to operate as compared to windows machines.

A meeting was scheduled with health care workers (medical assistants and data clerks), HMIS officer and DMO, to discuss the gaps and challenges identified with the existing system through the baseline audit, and the proposed evidence-based practice for malaria information system. There was an orientation and feedback session based on the proposed system.

During the discussion and orientation, possible challenges were explored to the use of EDC and the strategies and resources required to address them. A large concern was regarding the battery lifespan of the devices and the additional workload required to concurrently implement both paper and electronic systems.

Specific attention was given to the facility data clerks to ensure they understood how to conduct the data collection, and once completed, be able to send all the collected outpatient data (not only malaria patients) for their respective facilities to the central repository housed at the district health office of which the HMIS officer had administrative rights. Specific users such as the data clerks, DMO, were given access to the server and had some full control rights. The project team were assigned read only privileges to view the data only and monitor the data as it comes in. In addition, data cleaning scripts and report generating scripts were written to assist and automate the monthly report for each facility, as this was previously manually tallied by the data clerks on papers and transported via vehicle to the DHO office for data entry in their system.

The steps followed when coming up with the GRiP strategy were essential as they would help highlight the steps required in order to have a successful implementation program that is acceptable by everyone. The whole team specifically the users proposed the adoption of a mobile based EDC using the ODK application.

\section{Phase 3: Follow up audit post implementation of change strategy}

After implementation of the data collection tools, a follow up audit of the health facilities was made. Data entered into the local server was checked for various parameters as assessed in the baseline audit.

Phase 4: End of survey assessment 
A questionnaire for EDC assessment was developed which was used at the end of the survey to document the experience of users of the system.

\section{Results}

We found out that the current system of data capture involves the usage of registers in all the facilities. There are a number of registers the Malawi government through the Ministry of Health developed and allocated to all the health facilities. Some of them include the out-patient (OPD) department, antimalarial, laboratory, underfive and maternity registers. Since our main interest was malaria, we assessed the OPD, laboratory and antimalarial registers. During phase 1 stage when we assessed the health facilities, we discovered that the current paper-based system had problems which included:

1. Torn and lost papers within the registers

2. Illegible print (appendix 5)

3. Poor documentation by providers

4. Use of multiple registers to capture data of an individual

5. Filled up registers occupied a lot of space in the facilities reducing operational capacity (appendix 5)

Table 2 below summarizes comparisons between baseline and follow-up audits. It shows that there was no existence of electronic management information system (eMIS) at the beginning of the survey compared to the end. The malaria information system (MIS) used at all health centers did not have some necessary parameters, which include captured deaths from malaria and malaria treatment within 14 days.

Table 2: Baseline and Follow-Up audit outcomes 


\begin{tabular}{|c|c|c|c|c|c|c|c|c|}
\hline \multirow[t]{2}{*}{ Health Centre } & \multicolumn{2}{|c|}{ Majete } & \multicolumn{2}{|c|}{ Kakoma } & \multicolumn{2}{|c|}{ Kapichira } & \multicolumn{2}{|c|}{ Chapananga } \\
\hline & Base & End & Base & End & Base & End & Base & End \\
\hline $\begin{array}{l}\text { eMIS is used for quick access both } \\
\text { individual and aggregated data }\end{array}$ & No & Yes & No & Yes & No & Yes & No & Yes \\
\hline $\begin{array}{l}\text { Includes suspected malaria, tested } \\
\& \text { confirmed cases }\end{array}$ & Yes & Yes & Yes & Yes & Yes & Yes & Yes & Yes \\
\hline MIS includes deaths from malaria & No & No & No & No & No & No & No & No \\
\hline MIS includes patient location & Yes & Yes & Yes & Yes & Yes & Yes & Yes & Yes \\
\hline $\begin{array}{l}\text { MIS includes treatment (within } 14 \\
\text { days) }\end{array}$ & No & Yes & No & Yes & No & Yes & No & Yes \\
\hline $\begin{array}{l}\text { MIS includes malaria treatment and } \\
\text { dose }\end{array}$ & Yes & Yes & Yes & Yes & Yes & Yes & Yes & Yes \\
\hline $\begin{array}{l}\text { Roles, responsibilities, training } \\
\text { manuals \& modules available }\end{array}$ & No & No & No & No & No & No & No & No \\
\hline
\end{tabular}

\section{Baseline and Follow-up audits}

Figure 1 shows results for data completeness and consistency at baseline and follow up audits for 25 randomly selected records in the four health facilities. During the baseline audit, Kakoma was the only health center that had data completeness and consistency at 100\%. Majete 1 and Kapichira had data consistency and completeness both above $80 \%$. Chapananga had the least percentage in data consistency, $50 \%$ at baseline but attained $100 \%$ after the audit.

Tables 3 below summarizes the Grip matrix, involving identification of the barriers, strategies and resources used to reach the intended outcome. 
Table 3

GRIP matrix

\begin{tabular}{|llll|}
\hline Barrier & Strategy & Resources & Outcomes \\
\hline $\begin{array}{l}\text { Lack of support } \\
\text { from DHO }\end{array}$ & $\begin{array}{l}\text { Meetings and discussions; } \\
\text { potential benefits of involvement; } \\
\text { pioneering district in the country }\end{array}$ & $\begin{array}{l}\text { Transport; EDC } \\
\text { demonstration }\end{array}$ & $\begin{array}{l}\text { Adoption of piloting } \\
\text { EDC } \\
\text { implementation in } \\
\text { health facilities in } \\
\text { the district }\end{array}$ \\
$\begin{array}{l}\text { Reluctance for HF } \\
\text { data personnel to } \\
\begin{array}{l}\text { use EDC } \\
\text { (additional }\end{array}\end{array}$ & $\begin{array}{l}\text { Engagement of DHO and health } \\
\text { facility managers during baseline } \\
\text { and orientation; pioneering } \\
\text { facilities in the country }\end{array}$ & Orientation meetings & $\begin{array}{l}\text { Electronic data } \\
\text { collected and sent } \\
\text { to server }\end{array}$ \\
$\begin{array}{l}\text { Lack of technical } \\
\text { capacity to } \\
\text { implement EDC by } \\
\text { facility data } \\
\text { personnel }\end{array}$ & $\begin{array}{l}\text { Orientation and training for EDC; } \\
\text { involvement in the design of the } \\
\text { forms; technical support where } \\
\text { needed }\end{array}$ & $\begin{array}{l}\text { Provision of electronic } \\
\text { tablets; simplified } \\
\text { electronic forms; } \\
\text { training and }\end{array}$ & $\begin{array}{l}\text { Electronic data } \\
\text { collected and sent } \\
\text { to server }\end{array}$ \\
\hline
\end{tabular}

\section{Follow-up interview findings}

Users from all the facilities indicated that the EDC system was easy to use together with the use of electronic tablets and it made their work easier. They further established that the EDC improved their productivity and quality of care compared to the paper-based system. There were different views among users on whether the solutions that were developed and implemented met the intent of the promise. Some agreed with others highlighting that there were a number of changes made on the ODK form. Advantages that were mentioned by the users concerning EDC include:

1. Easy accessibility of data

2. Fast

3. Easy storage and management of data

4. Easy to transmit and send data

Despite this, the following disadvantages were mentioned:

1. The system required power supply, this posing a challenge at sites which had no access to the national grid.

2. Errors were noted in the ODK ranging from wrong spellings of villages/medication, improper dosages of some medications. These occurred when creating the ODK form.

3. Possible theft of the devices

4. Difficult to send electronic data to district health office because of lack of phone airtime needed for a wireless connection

5. Could not access the data to compile reports

\section{Discussion}


From our knowledge, this quality improvement project in rural Malawi is the first to use electronic devices in the form of tablets to validate the use of electronic data capturing on routinely collected health facility data for malaria and other notable conditions as an innovative approach for disease surveillance at sub-district level. The conditions of the location selected for this implementation project are comparable to other malaria endemic countries, and are characterized by being LMIC, dependent on subsistence farming, with reduced access to road networks, infrastructure, running water, electricity, etc.

Information systems, particularly at point of care, need to be simple and sustainable and not overburden health delivery staff. This study demonstrated that HCWs were able to reliably record and transmit data through an electronic reporting platform in this scenario, EDC using ODK on tablet software. With the use of EDC we were able to capture and incorporate some essential parameters that had not been developed and incorporated in the paper-based registers. Completeness and consistency of data in EDC was markedly better compared to the poor levels observed using the paper-based system. This could be attributed to the fact that manual entry of the paper-based registers, may have resulted in some reports being lost in the process, mix-up of entries as data clerks are responsible for handling multiple registers or no entry at all into the database due to other problems such as lack of stationery. Similarly, use of multiple registers to capture a single entry could have contributed to mix-up of entries as a wrong result could be given to a patient. Having many forms and registers being handled by a single HCW may contribute to having fatigue and loss of focus thereby resulting in having inaccurate data and illegible writings as seen in most of the health facilities. Furthermore, this can complicate the situation as it cannot be clearly established whether what has been reported reciprocates to what the HCW does.

Use of the EDC system in the current deployment eliminated the need for paper records by having all the required indicators pre-programmed into electronic forms and inserted into the ODK using Samsung tablet devices. It was easy for all HCWs to ensure that standard protocols are followed and the system itself was designed in a way as to not miss any field. This design facilitated complete records and proper storage. This system further provided an opportunity for remote quality control, as the data once collected could then be transferred from the remote server where other checks could be done to ensure consistency and completeness. The need to have EDC would also assist in reducing the problem of storage of records. In general, space that would be useful for service provision in rural health facilities could be turned into storage for filled up registers, so with EDC this problem could be overcome.

In the implementation effort reported here, EDC showed many attractive features and was highly regarded by HCWs in the field as a useful tool to increase their efficiency, and by supervisors as a great resource for quality control. Despite the achievements brought through the introduction of EDC, challenges of this system were also observed. Firstly, this system is effective mostly at sites where there is a reliable source of power to charge the tablets. Some facilities however did not have electricity as a result managing the devices was somehow difficult and the team had to find other means to avoid them shutting down. This situation is more complex in hard to reach areas where personnel need to travel long distances to get to a point where they can have access to power and it is typical for most rural areas in LMIC. For a program of this nature, access to power is very important for successful implementation. Possibly, cheaper forms of power such as solar electricity could be the solution for rural areas in order to address such problems. 
Another challenge raised was that devices are mostly prone to theft. There is a greater need to safeguard these devices otherwise implementation of the program would not be a success once these devices have gone missing. Typically, there is need to find ways of securing them mostly when they are not in use with means of tracing them. Since it was a pilot, one of the concerns that was also raised was the addition of workload as a result of adding EDC together with the paper based system. HCWs had to switch between use of registers and EDC so that data was ably captured using both data capture methods. There is also the problem of high turnover at most government facilities so a concern was raised that there would be need for orientation for new staff who would be responsible for data entry. For a successful project and sustainability of the implementation program, there is need for continuous engagement and orientation of all stakeholders, which at times can prove to be costly (19).

Facilities also mentioned that they experienced challenges with airtime to send data to the server, they would rely on finding other means of transport to the $\mathrm{DHO}$ so that data reaches the server. Through this discovery, it enabled us to discuss with the teams involved on better ways of mitigating these problems. There is need to find the best ways possible for the data to reach the server. This could require investing into the provision of adequate airtime so that data transmission should not be affected. As described by Proctor et al, adoption of an intervention best resides from the perspective of the provider (19), in this case, engaging the stakeholders was of great significance to the solutions of the problem. Facilities also were concerned that data being collected was not easy for them to access once sent to the DHO. This problem was due to the fact that access to the server was only limited to the administrator. It can be easily resolved by providing access to other service providers such as the data clerks and medical assistants from the facilities to have access to the server's URL.

The outcome measurements of the project met the expectations to the stakeholders in terms of accuracy, completeness, timeliness, transmission, and use of real-time data for feedback and support of HCWs in a remote setting in Malawi. Implementation of a system such as EDC using ODK on a larger scale, such as a whole district level, is therefore warranted and needs to be implemented in Malawi. This could be a reliable indicator of the feasibility of the system in those that would be using it and can be a determining factor for the scalability of this innovation. Some recommendations that were made included that refresher trainings should happen and that health facility in-charges should be involved when developing the ODK form to minimize errors. There is also need for proper planning for the project together with the district management team on how best EDC can be executed at a large scale. Since it is not yet adopted for use by the government at its facilities, the need of extra human resource such as additional data clerks to minimize the workload incurred with the introduction of EDC needs to be addressed. For proper continuity there is need for a proper timeframe to be put in place for the large scale pilot, results need to be discussed and disseminated with all the key stakeholders, including policy makers. There is also need for availability of devices that are working properly so as to avoid disruptions in data collection.

\section{Conclusion}

This work demonstrates that using mobile technologies such as EDC using the ODK platform in the capture and reporting of health facility data in remote locations of LMIC provides timely, complete and high quality data for malaria and other common conditions. Quality, complete and timely data collection by health workers

Page $11 / 15$ 
in a remote setting in Malawi is achievable and such data can be transmitted, aggregated and used by HCWs and the district to improve their service delivery.

\section{Abbreviations}

AL: Artemisinin-lumefantrine; DHIS: District health information system; DMO: District medical officer; eCRF: electronic case report form; GRiP: Getting research into practice; GTS: Global Technical Strategy for Malaria; HCW: Health care worker; HMIS: Health management information system; JBI: Johanne Briggs Institute; LMIC: Low and middle income countries; MIS: Malaria information system; MMP: Majete Malaria Project; mRDT: Malaria rapid diagnostic test; MWRP: Majete Wildlife Reserve Perimeter; ODK: Open data kit; OPD: Outpatient department; PACES: Practical Application of Clinical Evidence System; SSA: Sub Saharan Africa; URL: Uniform resource locator; WHO: World Health Organization; XML: Extensible Markup Language

\section{Declarations}

\section{Ethics approval and consent to participate}

Not applicable.

\section{Consent for publication}

Not applicable.

\section{Availability of data and materials}

The dataset is accessible at the corresponding author upon a reasonable request.

\section{Conflict of interest}

Tinashe Tizifa, William Nkhono, Spencer Mtengula, Sandeep Moola, Michele van Vugt, Zachary Munn, and Alinune Kabaghe declare that they have no conflict of interest.

\section{Funding}

This work was supported by the Majete Malaria Project, with funding from Dioraphte Foundation, the Netherlands. The project provided both technical and logistical support. The content is solely the responsibility of the authors and does not necessarily represent the official views of the funders.

\section{Authors' contributions}

Research idea and study design: TT, ANK AND WN; Data acquisition: TT, WN, SM and ANK; Data analysis and Interpretation: TT, ANK and WN; Supervision and Mentoring: ANK and MvV. TT wrote the first draft of the manuscript. Each author contributed important intellectual content during manuscript revision and writing of the final manuscript. All authors reviewed and approved the final manuscript.

\section{Acknowledgements}


We would like to thank JBI, University of Adelaide for providing technical guidance and a scholarship for the JBI fellowship. We are grateful to Professor Kamija Phiri co-PI of the Majete Malaria Project for reviewing this manuscript. We also would like to thank Sandeep Moola for providing some literature on evidence implementation. Finally, the Ministry of Health, Chikhwawa district health office for making it possible for us to conduct the pilot within the district.

\section{Authors information (Optional)}

${ }^{1}$ Center for Tropical Medicine and Travel Medicine, Department of Infectious Diseases, Division of Internal Medicine, University of Amsterdam, University Medical Center, Amsterdam, The Netherlands.

${ }^{2}$ Training and Research Unit of Excellence (TRUE), School of Public Health and Family Medicine, College of Medicine, University of Malawi, Blantyre, Malawi.

${ }^{3} \mathrm{JBI}$, Faculty of Health and Medical Sciences, University of Adelaide, Adelaide, Australia.

\section{Author's emails}

Tinashe A.Tizifa - t.tizifa@amsterdamumc.nl

William Nkhono - williamnkhono@hotmail.com

Spencer Mtengula - smtengula@medcol.mw

Michele van Vugt - m.vanvugt@amsterdamumc.nl

Zachary Munn - zachary.munn@adelaide.edu.au

Alinune N. Kabaghe - akabaghe@medcol.mw

\section{References}

1. World malaria report 2018. 2018.

2. Alemu K, Worku A, Berhane Y. Malaria infection has spatial, temporal, and spatiotemporal heterogeneity in unstable malaria transmission areas in northwest ethiopia. PLoS One. 2013;8(11):1-10.

3. Global technical strategy for malaria 2016-2030. 2016;

4. Rosewell A, Makita L, Muscatello D, John LN, Bieb S, Hutton R, et al. Health information system strengthening and malaria elimination in Papua New Guinea. Malar J. 2017;16(1):1-10.

5. Ohrt C, Roberts KW, Sturrock HJW, Wegbreit J, Lee BY, Gosling RD. Information Systems to Support Surveillance for Malaria Elimination. Am J Trop Med Hyg. 2015;93(1):145-52.

6. Yukich JO, Butts J, Miles M, Berhane Y, Nahusenay H, Malone JL, et al. A description of malaria sentinel surveillance: A case study in Oromia Regional State, Ethiopia. Malar J. 2014;13(1):1-13.

7. Roca-feltrer A, Lalloo DG, Phiri K, Terlouw DJ. Short Report: Rolling Malaria Indicator Surveys ( rMIS ): A Potential District-Level Malaria Monitoring and Evaluation ( $M \& E$ ) Tool for Program Managers. 2012;86(1):96-8. 
8. Afrane YA, Zhou G, Githeko AK, Yan G. Utility of Health Facility-based Malaria Data for Malaria Surveillance. Schallig HDFH, editor. PLoS One [Internet]. 2013 Feb 13 [cited 2020 Aug 19];8(2):e54305. Available from: https://dx.plos.org/10.1371/journal.pone.0054305

9. Kabaghe AN, Phiri MD, Phiri KS, Van Vugt M. Challenges in implementing uncomplicated malaria treatment in children: A health facility survey in rural Malawi. Malar J [Internet]. 2017 Oct 18 [cited 2020 Oct 1];16(1). Available from: https://pubmed.ncbi.nlm.nih.gov/29047388/

10. Initiative M. PRESIDENT'S MALARIA INITIATIVE Malawi Malaria Operational Plan FY 2018 [Internet]. [cited 2019 Jul 24]. Available from: https://www.pmi.gov/docs/default-source/default-documentlibrary/malaria-operational-plans/fy-2018/fy-2018-malawi-malaria-operational-plan.pdf?sfvrsn=5

11. Porritt K, McArthur A, Lockwood C, Munn Z, editors. JBI Manual for Evidence Implementation [Internet]. JBI; 2020 [cited 2020 Oct 1]. Available from: https://wiki.jbi.global/display/JHEI

12. Al MA et. The ripple effect of evidence implementation: a descriptive evaluation of JBI's Evidence-based Clinical Fellowship Program. JBI Evidence Implementation.

13. Lizarondo, Lucylynn PhD, MPhysio, MPsych1; McArthur, Alexa RN, RM, MPHC, MClinSc2; Lockwood, Craig PhD1; Munn ZP. Facilitation of evidence implementation within a clinical fellowship program, International Journal of Evidence-Based Healthcare: S, 2020 - Volume Publish Ahead of Print - Issue 10.1097/XEB.0000000000000252, Doi:

14. Lockwood C, Stannard D, Jordan Z, Porritt K. The Joanna Briggs Institute clinical fellowship program. Int J Evid Based Healthc [Internet]. 2020 Mar 1 [cited 2020 Oct 1];18(1):1-4. Available from: http://journals.Iww.com/10.1097/XEB.0000000000000221

15. Kabaghe AN, Chipeta MG, Mccann RS, Phiri KS, Vugt V, Takken W, et al. Adaptive geostatistical sampling enables efficient identification of malaria hotspots in repeated cross-sectional surveys in rural Malawi. 2017;1-14.

16. Health Organization W. Disease surveillance for malaria control: an operational manual.

17. Guidelines for the Treatment of Malaria. Third Edition - World Health Organization - Google Books [Internet]. [cited 2020 Sep 9]. Available from: https://books.google.mw/books? $\mathrm{hl}=$ en\&lr=\&id=IVoODgAAQBAJ\&oi=fnd\&pg=PP1\&dq=1.+The+World+Health+Organization+ (WHO).+Guidelines+for+the+treatment+of+malaria+-+3rd+edition.+2015.+WHO+Press:+Geneva.+ (Level+5)\&ots=9Trg6oQ2gJ\&sig=zVdiQqbmQsvuSbHIEBMpmeNQ53k\&redir_esc=y\#v=onepage\&q\&f=false

18. Macharia Paul, Samabi Betsy, Dunbar Mathew, Wamuti Beatrice, Maingi Petrer, Felix Abuna, David Bukusi CP and FC. Using Open Data Kit in acluster Randomized Clinical Trial: The Assisted Partner Services Study. 2018;5(Biostec):247-52.

19. Proctor E, Silmere H, Raghavan R, Hovmand P, Aarons G, Bunger A, et al. Outcomes for implementation research: Conceptual distinctions, measurement challenges, and research agenda. Adm Policy Ment Heal Ment Heal Serv Res. 2011;38(2):65-76.

\section{Figures}




\section{Base and Endline Audit Results}

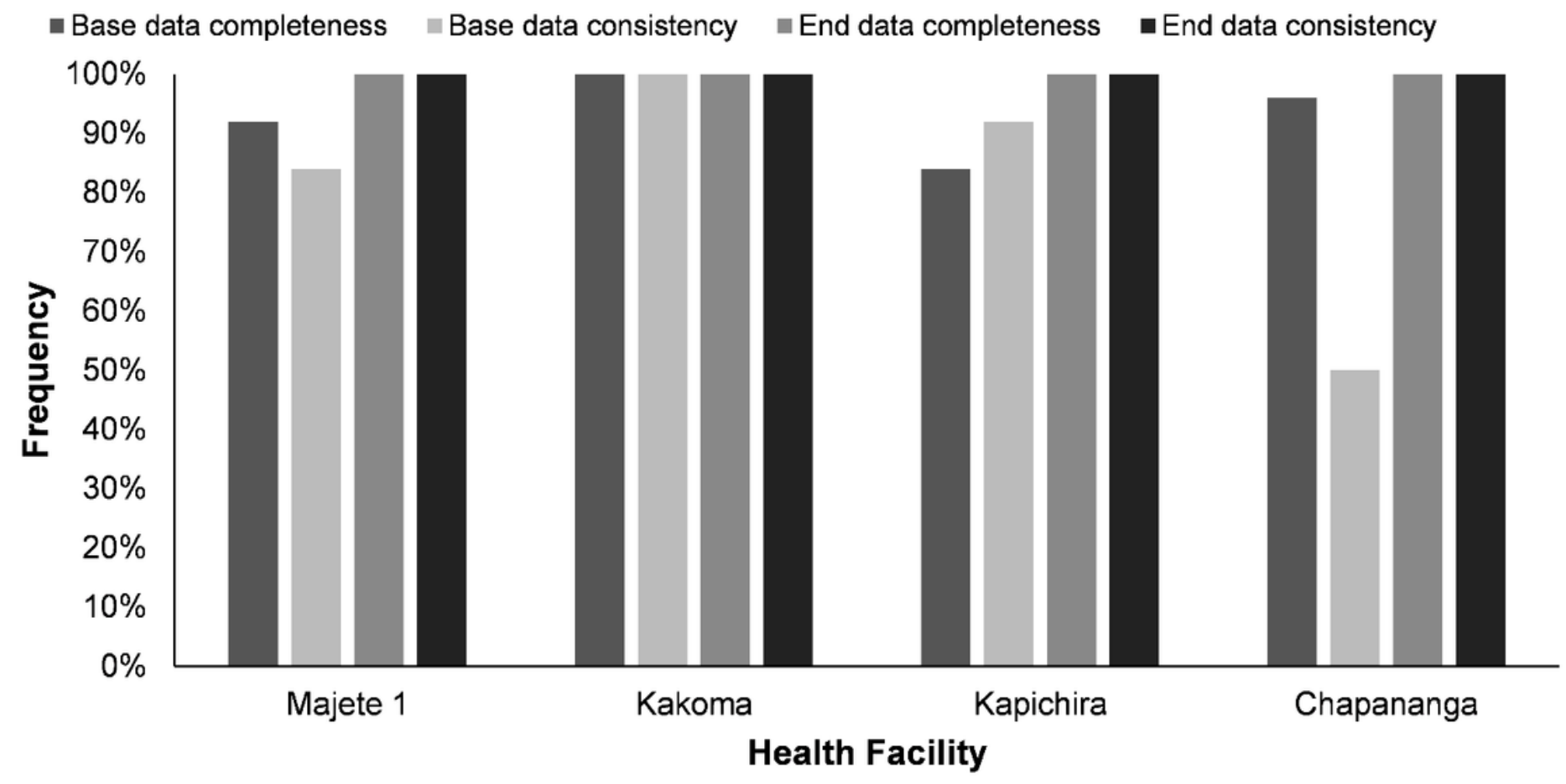

Figure 1

Compliance with best practice audit criteria in follow up audit compared to baseline audit (\%)

\section{Supplementary Files}

This is a list of supplementary files associated with this preprint. Click to download.

- APPENDICESFOREDCTTIZIFA.docx 